\title{
Two steps forward in treating spinal cord injury
}

Two studies presented at Neuroscience 2012 , the annual meeting of the Society for Neuroscience (13-17 October 2012; New Orleans, LA), reflect current advances in understanding and treating spinal cord injury (SCI). More than a quarter of a million people in the US live with SCIs, which can be debilitating because they limit movement or feeling, cause pain and lead to chronic health problems.

"While the damage of SCI can appear to be immediate and dramatic, the biological events that lead to extensive nerve and tissue damage are complex, and injuries evolve over time," said moderator Jacqueline Bresnahan (University of California, San Francisco), an expert on traumatic SCI, in a press conference. "Today researchers are finding ways to intervene in the cascade of molecular changes that follow SCI [and] finding new ways to treat and rehabilitate patients," she continued.

Brian Noga (University of Miami, FL) reported on the use of deep brain stimulation (DBS) to restore walking ability in cases of partial SCI. DBS is already used to treat other movement disorders, including Parkinson's disease, but has not been previously applied to treating SCI. In Noga's study, rats with partial SCIs received stimulation from an electrode implanted in the mesencephalic locomotor region of the brain. This DBS activated descending nerve fibers, improving both the speed and the distance that injured rats were able to travel. Some rats that had not been able to step forward after injury began walking again during the 10 -week study. These results indicate that DBS can reactivate nerve pathways that are intact but dysfunctional after partial SCI and may be useful for improving walking in people with chronic SCI.

Yang Teng (Harvard Medical School and Veterans Affairs Boston Healthcare System, Boston, MA) reported success using a different strategy to improve walking and reduce damage after SCI in rats: inhalation of carbon monoxide (CO). Rats were exposed to one of three doses of CO for 1 hour per day for 12 days. Those exposed to the highest dose of CO (500 ppm) showed

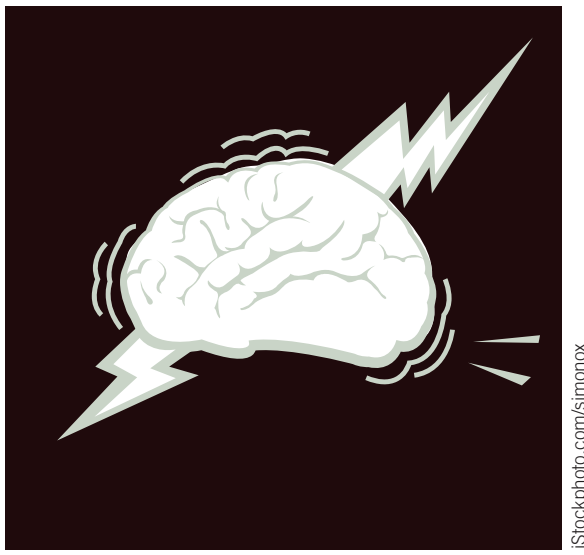

the greatest improvements in walking ability and in recovery from SCI as measured by nerve lesion volume and survival of motor neurons. These neuroprotective effects of $\mathrm{CO}$ exposure resulted from the interference of CO with the body's inflammatory response to SCI. Teng and colleagues suggest that $\mathrm{CO}$ inhalation may represent a new neuroprotective treatment for traumatic SCI.

Monica Harrington

\section{SLEEPY BEES SHOULD PULL OVER FOR DIRECTIONS}

It is well documented that sleep has a supportive effect on memory consolidation in animals. In fact, research has shown that specific types of memory consolidation, in humans, are linked to different stages of sleep. Conversely, there is also evidence that certain kinds of memory consolidation may be independent of sleep.

In a paper published in the Journal of Experimental Biology $(215,3981-3988 ; 2012)$, Randolf Menzel and his colleagues at the Free University of Berlin (Germany) study honey bees to explore whether learning during navigation leads to sleep-dependent memory consolidation. Menzel and his team first characterized how honey bees find their way home after being captured and released in unfamiliar territory (forced navigation task). Select bees were outfitted with radio frequency identification devices (RFIDs), and their inbound and outbound flights were monitored as well as their homing and hive behavior, including their rest during the day and sleep at night. RFID-marked bees behaved no differently than unmarked bees, both inside and outside the hive.

The team found that after the forced navigation task, the bees slept longer during the first part of the night, but their other foraging behavior remained unchanged. To test whether this increase in sleep might reflect some sort of learning or memory process, the researchers decided to disturb the slumber of select bees by placing them in a box that was gently agitated for 8 hours during the night.

The next day, both sleep-deprived and rested bees underwent another forced navigation task, in which they were released $600 \mathrm{~m}$ from the hive in a novel location. Both groups performed equally well on this task, indicating that lack of sleep did not affect the bees' navigational learning process. But after a second night of disturbed sleep followed by the same forced navigation task, sleepy honey bees were just as likely to get lost as they had been the day before, whereas their well-rested counterparts performed dramatically better, cutting their odds of getting lost by half. Interestingly, the time it took to successfully return home did not vary between groups for the first and second homing flights. This result implies that sleep deprivation did not disrupt the bees' ability to access the memory used during the first homing flight but rather interfered with the consolidation of the newly acquired navigational memory.

In short, sleepy honey bees have a harder time remembering lessons learned the day before than their well-rested hivemates. 